Kong. Res. J. 4(2): 173-177, 2017

ISSN 2349-2694

Kongunadu Arts and Science College, Coimbatore.

\title{
ALLELOPATHIC INFLUENCE OF SOME WEED RESIDUES ON GROWTH AND DEVELOPMENTAL CHANGES OF GREEN GRAM (VIGNA RADIATA (L.) WILCZEK)
}

\author{
Kavitha, D., J. Prabhakaran* and K. Arumugam \\ Department of Botany, Annamalai University, Annamalai Nagar-608 002. \\ *E.mail: drprabha2006@gmail.com
}

\begin{abstract}
The term allelopathy refers to the detrimental effects of higher plants of one species (the donor) on the germination, growth, or development of another species (the recipient). In the present studyallelopathic influence of weed species, Echinochloa colona (L.) Link, (Poaceae), Cleome viscosa L. (Capparidaceae) and Ammania baccifera L. (Lythraceae) on green gram (Vigna radiata (L.) Wilczek were investigated. The individual and combined residues of three weeds wereincorporated to the soil at the quantities of 0,1,2,3 and $4 \%(\mathrm{w} / \mathrm{w})$ in the plots and the germination, seedling growth dry weight of green gram were assessed. The results showed that all the concentrations of combined weed residues exhibited higher degree of inhibitory effects than the individual weed residues in all the parameters employed in the study except at $1 \%$ of $C$. dactylon, where insignificant growth promotion observed. The percentage of inhibitory effects of weed residues increases with increasing the magnitude of the residues. The degree of reduction percentage of all the growth parameters was concentration dependent.Among the three weeds, A. baccifera had more retarding effects on the growth of green gram and the order of inhibitory effect of three weed was $A$. baccifera, C. viscosa and E. colona.
\end{abstract}

Keywords: Allelopathy, A. baccifera, C. viscosa, E. colona, green gram, Weed residues.

\section{INTRODUCTION}

Allelopathy is recognized as an important ecological mechanism which influences plant dominance, succession and formation of plant communities, vegetation and crop productivity. It has been related to the problems with weed: crop interference. Weeds cause greater losses in crop yields than either insects or plant diseases. The weeds reduce the crop yields through (a) allelopathy, i.e., release of inhibitors from seeds, living plants and plant residues, (b) competition for growth resources (light, nutrients, water and space) with crops and (c) acting as an alternate host for insects and disease organisms. The decomposition of plant residues adds the largest quantity of allelochemicals to the soil. At plant death, materials compartmentalized in cells are released into the environment. The nature of the plant residues, the soil type are important pre requisite for decomposition. As the roots grow through the soil, at some points they may get in touch with decaying plant residues and are impacted by allelochemicals. The decomposition of plant residues potentially provides the largest quantity of allelochemicals that may be added to the rhizosphere. Patrick et al. (1964) reported that depending on the decomposing conditions, substances highly toxic, non-toxic or stimulatory to plants might be formed during the decomposition of similar plant residues.Different weed species differ widely in their ability to produce allelopathic effects (Hamayun et al., 2005). Different parts of same weed also differ in their ability to produce allelopathic effects on germination and growth of crop plants. Some parts are inhibitorier than others (Tanveer et al., 2008). A number of studies have shown that allelochemicals release into the soil from residues of weeds, thus affecting the growth of crop plants (Kumar et al., 2009). Furthermore, many allelopathic plants incorporated in soil are known to inhibit the growth of other plants (Rajashekhara et al., 2007).

Green gram (Vigna radiata (L.) Wilczek), is one of the important pulse crop cultivated as intercrop along with rice and in the follow field of rice crop in Cuddalore District of Tamil Nadu India. Therefore, the present investigation, three common dominant weed species of paddy fields, namely, Echinochloa colona (L.) Link, (Poaceae), Cleome viscose L. and Ammania baccifera L. (Lythraceae) were selected to evaluate their allelopathic potential on germination and seedling growth of green gram (Vigna radiata (L.) Wilczek).

\section{MATERIALS AND METHODS}

Weed species were collected from post harvest paddy fields of Cuddalore District, Tamil Nadu and various quantities their residues were prepared from shade dried whole plant of the weeds 
and also the equal quantity of three weed residues were mixed together for combined weed residues. Field experiments were conducted in the split plots $(0.75 \times 0.75 \mathrm{~m})$ on the basis of Randomized Complete Block Design with three replicates. The weed residues were incorporated to the soil at the quantities of $0,1,2,3$ and $4 \%(\mathrm{w} / \mathrm{w})$ in the plots. The residue incorporated soil was allowed for three weeks afterwards the green gram cv.ADT-3 seeds were sown.

The parameters employed in the present studies are germination percentage,seedling length, root, stem and leaf biomass production of green gram were recorded at 15, 30, 45 and 60day old seedlings. Obtained data were analysed by ANOVA followed by Tuke'sMultiple Range Test at 5\% probability level.

\section{RESULTS AND DISCUSSION}

Allelopathic influence of weed residues showed adverse effects on the seed germination (Fig.1), growth and pigment contents of green gram. The individual and combined residues of all the three weed species destructively influenced on the germination and growth of green gram except at $1 \%$ of residue of E. colona, where a slight non-significant stimulatory effects observed.Similar results were noticed by number of researchers. Quayyam et al. (2000) reported that the Cyperus rotundus aqueous extracts and leachate of leaves and tubers significantly reduced the germination and seedling growth of rice. The leachate of Echinochloa colonum inhibited the germination and seedling growth of onion, radish and knolkhol (Challa and Ravindra,1998), Cynodon dactyton, Cyperus rotundus and other four weeds are adversely inhibited the seed germination of and growth of tomato (Sannigrahi and Chakrabortty, 2005). The root and shoot extracts of Cyperus rotundus decreased the seedling growth of rice with the reduction in root and shoot length, fading of leaves and curling of leaf tips (Bartariya et al, 2005). At higher concentrations of three weed residues showed maximum adverse effect on germination of green gram.The inhibition on seed germination may be due to the presence of high amount of allelochemicals in the weed residues.

Morphometric measurements viz., seedling length, root, stem and leaf dry biomass of green gram crop were determined at four stages. A glimpse at tables-1-5 also reflected the inhibitory effects on germination. Apart from affecting germination, the root development, which plays a contributory role in plant growth, was also depressed. Understandably, a poorly development root system is disadvantageous to the emerging seedlings. This is exactly the trend emerging from the data where 16.8 to $65.6 \%$ of inhibition was evident. Allelochemicals decreased elongation, expansion and division of cells which are growth prerequisite (Qasem and Hill, 1989) Also, allelochemicals inhibit absorption of ions (Dos Santosh et al., 2004) and therefore, resulted in arrested growth (Venkateshwarlu, 2001). One of the suggested explanation for disruption of seedling growth and development during allelopathy stress is modification in mitochondrial respiration leading to decreased supply of ATP for all energy demanding processes .The reduction of plant growth in the presence of allelochemicals is associated with the strong inhibition of mitosis or/and disruption of the structure of organelles e.g. nuclei and mitochondria (Gniazdowska and Bogatek, 2005).

All the concentrations of combined weed residues exhibited higher inhibitory effects than the individual weed residues in all the parameters employed in the study in all the four growth stages of the test crop. The percentage of inhibitory effects of weed residues increases with increasing the magnitude of the residues. The degree of reduction percentage was concentration dependent. The statistical analysis of all the parameters tested in the present investigation showed that significance effect of individual and combined extracts of three weed species on germination, growth, biochemical constituents and productivity of green gram.

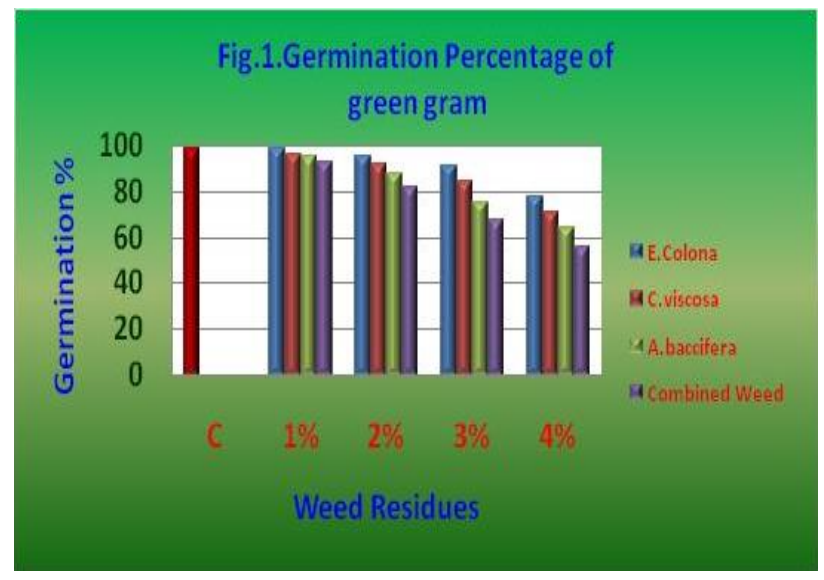

Among the three weeds, A. baccifera had more retarding effect on the growth of green gram and the order of inhibitory effect of weed was A.baccifera, C.viscosa and E.colona. Hence it can be concluded that all the three weed species strongly exerted their negative allelopathic potential on the germination, growth and dry biomass of green gram (Vigna radiata (L.) Wilczek) cv-ADT-3. 
Table 1. Root length (cm/plant) of Green gram plants treated with various concentrations of individual and combined weed residues.

\begin{tabular}{|c|c|c|c|c|c|c|c|c|c|c|c|c|c|c|c|c|}
\hline \multirow{3}{*}{$\begin{array}{c}\text { Weed } \\
\text { Residues } \\
\text { (\%) }\end{array}$} & \multicolumn{4}{|c|}{ E. colona } & \multicolumn{4}{|c|}{ C. viscosa } & \multicolumn{4}{|c|}{ A. baccifera } & \multicolumn{4}{|c|}{ Combined weeds } \\
\hline & \multicolumn{4}{|c|}{ DAS } & \multicolumn{4}{|c|}{ DAS } & \multicolumn{4}{|c|}{ DAS } & \multicolumn{4}{|c|}{ DAS } \\
\hline & 15 & 30 & 45 & 60 & 15 & 30 & 45 & 60 & 15 & 30 & 45 & 60 & 15 & 30 & 45 & 60 \\
\hline $\mathrm{C}$ & $7.5 a$ & $8.9 b$ & $16.0 \mathrm{~b}$ & $21.4 \mathrm{a}$ & $7.5 \mathrm{a}$ & $8.9 a$ & $16 a$ & $21.4 a$ & $7.5 \mathrm{a}$ & $8.9 a$ & $16 a$ & $21.4 \mathrm{a}$ & $7.5 \mathrm{a}$ & $8.9 a$ & $16 a$ & $21.4 a$ \\
\hline 1 & $7.8 \mathrm{a}$ & $9.4 \mathrm{a}$ & $17.1 \mathrm{a}$ & $22.2 \mathrm{a}$ & $7.2 \mathrm{a}$ & $8.6 a$ & $15.6 a$ & $20.2 b$ & $6.8 b$ & $8.4 \mathrm{~b}$ & $15.3 \mathrm{~b}$ & $20.6 b$ & $6.7 b$ & $8.2 b$ & $15.5 a$ & $19.9 b$ \\
\hline 2 & $7.2 \mathrm{~b}$ & $8.6 \mathrm{~b}$ & $15.2 b$ & $19.4 \mathrm{~b}$ & $6.7 b$ & $7.9 b$ & $14.7 \mathrm{~b}$ & $18.8 \mathrm{~b}$ & $6.4 \mathrm{~b}$ & $7.6 \mathrm{c}$ & $14.3 \mathrm{c}$ & $18.3 \mathrm{c}$ & $6.2 \mathrm{~b}$ & $7.6 \mathrm{~b}$ & $13.7 b$ & $17.6 \mathrm{c}$ \\
\hline 3 & $6.4 \mathrm{c}$ & $7.9 \mathrm{c}$ & $13.7 \mathrm{c}$ & $18.5 \mathrm{c}$ & $6.1 \mathrm{c}$ & $7.4 \mathrm{c}$ & $13.0 \mathrm{c}$ & $17.6 \mathrm{c}$ & $5.8 c$ & $7.1 d$ & $12.7 \mathrm{~d}$ & $17.0 \mathrm{~d}$ & $5.4 \mathrm{c}$ & $6.9 c$ & $11.8 \mathrm{c}$ & $16.3 \mathrm{~d}$ \\
\hline 4 & $6.2 c$ & $7.6 \mathrm{~d}$ & $13.0 \mathrm{c}$ & $17.4 \mathrm{~d}$ & $5.7 \mathrm{c}$ & $7.0 \mathrm{c}$ & $11.6 \mathrm{~d}$ & $16.5 \mathrm{e}$ & $5.4 \mathrm{c}$ & $6.8 \mathrm{~d}$ & $11.2 \mathrm{c}$ & $15.6 \mathrm{e}$ & $5.0 \mathrm{c}$ & $6.2 \mathrm{~d}$ & $10.2 \mathrm{~d}$ & $13.6 \mathrm{e}$ \\
\hline
\end{tabular}

Mean with different alphabets in a column differed significantly as per Tukey's Multiple Range Test (TMRT) $(\mathrm{P}<0.05)$.

Table 2. Shoot length (cm/plant) of Green gram plants treated with various concentrations of individual and combined weed residues.

\begin{tabular}{|c|c|c|c|c|c|c|c|c|c|c|c|c|c|c|c|c|}
\hline \multirow{3}{*}{$\begin{array}{c}\text { Weed } \\
\text { Residues } \\
\text { (\%) }\end{array}$} & \multicolumn{4}{|c|}{ E. colona } & \multicolumn{4}{|c|}{ C. viscosa } & \multicolumn{4}{|c|}{ A. baccifera } & \multicolumn{4}{|c|}{ Combined weeds } \\
\hline & \multicolumn{4}{|c|}{ DAS } & \multicolumn{4}{|c|}{ DAS } & \multicolumn{4}{|c|}{ DAS } & \multicolumn{4}{|c|}{ DAS } \\
\hline & 15 & 30 & 45 & 60 & 15 & 30 & 45 & 60 & 15 & 30 & 45 & 60 & 15 & 30 & 45 & 60 \\
\hline $\mathrm{C}$ & $15.3 \mathrm{~b}$ & $20.3 b$ & $23.8 \mathrm{~b}$ & $27.2 \mathrm{~b}$ & $15.3 a$ & $20.3 a$ & $23.8 \mathrm{a}$ & $27.2 \mathrm{a}$ & $15.3 a$ & $20.3 a$ & $23.8 \mathrm{a}$ & $27.2 \mathrm{a}$ & $15.3 a$ & $20.3 a$ & $23.8 \mathrm{a}$ & $27.2 \mathrm{a}$ \\
\hline 1 & $16.2 \mathrm{a}$ & $21.7 \mathrm{a}$ & $25.3 a$ & $28.4 a$ & $14.8 \mathrm{a}$ & $19.2 b$ & $22.7 b$ & $25.4 \mathrm{~b}$ & $14.5 a$ & $18.0 \mathrm{~b}$ & $21.8 b$ & $24.2 \mathrm{~b}$ & $13.5 b$ & $17.5 \mathrm{~b}$ & $21.3 b$ & $23.9 b$ \\
\hline 2 & $14.1 \mathrm{c}$ & $19.5 b$ & $22.8 \mathrm{c}$ & $26.2 \mathrm{c}$ & $13.9 \mathrm{~b}$ & $18.1 \mathrm{c}$ & $19.8 c$ & $22.5 \mathrm{c}$ & $12.0 \mathrm{c}$ & $17.2 \mathrm{~b}$ & $18.3 \mathrm{c}$ & $21.2 \mathrm{c}$ & $10.8 \mathrm{c}$ & $16.1 \mathrm{c}$ & $16.1 \mathrm{c}$ & $20.7 \mathrm{c}$ \\
\hline 3 & $13.5 \mathrm{c}$ & $18.7 \mathrm{c}$ & $21.1 \mathrm{~d}$ & $24.2 \mathrm{~d}$ & $11.5 \mathrm{c}$ & $17.2 \mathrm{~d}$ & $19.0 \mathrm{~d}$ & $20.4 d$ & $10.4 \mathrm{~d}$ & $15.1 \mathrm{c}$ & $15.8 d$ & $19.7 d$ & $9.3 \mathrm{~d}$ & $13.2 \mathrm{~d}$ & $14.3 \mathrm{~d}$ & $17.5 d$ \\
\hline 4 & $12.0 \mathrm{~d}$ & $16.7 \mathrm{~d}$ & $19.19 \mathrm{e}$ & $21.7 \mathrm{e}$ & $10.1 d$ & $15.3 \mathrm{e}$ & $16.9 \mathrm{e}$ & $18.7 \mathrm{~d}$ & $8.3 \mathrm{e}$ & $13.0 \mathrm{~d}$ & $14.3 \mathrm{e}$ & $17.6 \mathrm{e}$ & $6.8 \mathrm{e}$ & $11.5 \mathrm{c}$ & $12.5 \mathrm{e}$ & $14.7 \mathrm{e}$ \\
\hline
\end{tabular}

Mean with different alphabets in a column differed significantly as per Tukey's Multiple Range Test (TMRT) $(\mathrm{P}<0.05)$. 
Table 3. Root Dry weight (g/plant) of Green gram plants treated with various concentrations of individual and combined weed residues.

\begin{tabular}{|c|c|c|c|c|c|c|c|c|c|c|c|c|c|c|c|c|}
\hline \multirow{3}{*}{$\begin{array}{c}\text { Weed } \\
\text { Residues (\%) }\end{array}$} & \multicolumn{4}{|c|}{$\begin{array}{c}\text { E. colona } \\
\text { DAS }\end{array}$} & \multirow{2}{*}{\multicolumn{4}{|c|}{$\begin{array}{c}\text { C. viscosa } \\
\text { DAS }\end{array}$}} & \multirow{2}{*}{\multicolumn{4}{|c|}{$\frac{\text { A. baccifera }}{\text { DAS }}$}} & \multirow{2}{*}{\multicolumn{4}{|c|}{$\begin{array}{c}\text { Combined weeds } \\
\text { DAS }\end{array}$}} \\
\hline & \multicolumn{4}{|c|}{ DAS } & & & & & & & & & & & & \\
\hline & 15 & 30 & 45 & 60 & 15 & 30 & 45 & 60 & 15 & 30 & 45 & 60 & 15 & 30 & 45 & 60 \\
\hline $\mathrm{C}$ & $0.050 \mathrm{~b}$ & $0.232 \mathrm{~b}$ & $0.623 a$ & $0.664 a$ & $0.050 \mathrm{a}$ & $0.232 \mathrm{a}$ & $0.623 a$ & $0.664 a$ & $0.050 \mathrm{a}$ & $0.232 \mathrm{a}$ & $0.623 a$ & $0.664 a$ & $0.050 \mathrm{a}$ & $0.232 \mathrm{a}$ & $0.623 a$ & $0.664 a$ \\
\hline 1 & $0.065 \mathrm{a}$ & $0.248 \mathrm{a}$ & $0.636 a$ & $0.673 a$ & $0.046 \mathrm{~b}$ & $0.215 b$ & $0.614 \mathrm{a}$ & $0.637 \mathrm{~b}$ & $0.043 \mathrm{~b}$ & $0.206 \mathrm{~b}$ & $0.059 \mathrm{~b}$ & $0.605 \mathrm{~b}$ & $0.041 \mathrm{~b}$ & $0.187 \mathrm{~b}$ & $0.567 \mathrm{~b}$ & $0.584 \mathrm{~b}$ \\
\hline 2 & $0.049 \mathrm{~b}$ & $0.225 c$ & $0.611 \mathrm{~b}$ & $0.626 \mathrm{~b}$ & $0.041 \mathrm{c}$ & $0.197 \mathrm{c}$ & $0.568 \mathrm{~b}$ & $0.603 c$ & $0.039 \mathrm{c}$ & $0.179 \mathrm{c}$ & $0.537 \mathrm{c}$ & $0.575 c$ & $0.037 c$ & $0.165 \mathrm{c}$ & $0.494 \mathrm{c}$ & $0.553 c$ \\
\hline 3 & $0.045 b$ & $0.209 \mathrm{~d}$ & $0.549 \mathrm{c}$ & $0.604 \mathrm{c}$ & $0.037 \mathrm{~d}$ & $0.178 \mathrm{~d}$ & $0.494 \mathrm{c}$ & $0.577 \mathrm{~d}$ & $0.035 \mathrm{~d}$ & $0.016 \mathrm{~d}$ & $0.467 \mathrm{~d}$ & $0.534 \mathrm{~d}$ & $0.033 \mathrm{~d}$ & $0.146 \mathrm{~d}$ & $0.424 \mathrm{~d}$ & $0.503 \mathrm{~d}$ \\
\hline 4 & $0.042 \mathrm{c}$ & $0.193 \mathrm{e}$ & $0.495 \mathrm{~d}$ & $0.578 \mathrm{~d}$ & $0.033 \mathrm{e}$ & $0.155 \mathrm{e}$ & $0.447 \mathrm{~d}$ & $0.505 \mathrm{e}$ & $0.029 \mathrm{e}$ & $0.141 \mathrm{e}$ & $0.414 \mathrm{e}$ & $0.465 \mathrm{e}$ & $0.027 \mathrm{e}$ & $0.124 \mathrm{e}$ & $0.364 \mathrm{e}$ & $0.427 \mathrm{e}$ \\
\hline
\end{tabular}

Mean with different alphabets in a column differed significantly as per Tukey's Multiple Range Test (TMRT) $(\mathrm{P}<0.05)$.

Table 4. Stem Dry weight (g/plant) of Green gram plants treated with various concentrations of individual and combined weed residues.

\begin{tabular}{|c|c|c|c|c|c|c|c|c|c|c|c|c|c|c|c|c|}
\hline \multirow{3}{*}{$\begin{array}{c}\text { Weed } \\
\text { Residues } \\
(\%)\end{array}$} & \multirow{2}{*}{\multicolumn{4}{|c|}{ E. colona }} & \multirow{2}{*}{\multicolumn{4}{|c|}{$\begin{array}{c}\text { C. viscosa } \\
\text { DAS }\end{array}$}} & \multirow{2}{*}{\multicolumn{4}{|c|}{ A. baccifera }} & \multicolumn{4}{|c|}{ Combined weeds } \\
\hline & & & & & & & & & & & & & & & & \\
\hline & 15 & 30 & 45 & 60 & 15 & 30 & 45 & 60 & 15 & 30 & 45 & 60 & 15 & 30 & 45 & 60 \\
\hline $\mathrm{C}$ & $0.035 a$ & $0.333 \mathrm{~b}$ & $0.907 \mathrm{~b}$ & $1.531 \mathrm{~b}$ & $0.035 b$ & $0.333 a$ & $0.907 a$ & $1.531 \mathrm{a}$ & $0.035 a$ & $0.333 a$ & $0.907 a$ & $1.531 \mathrm{a}$ & $0.035 a$ & $0.333 a$ & $0.907 a$ & $1.531 \mathrm{a}$ \\
\hline 1 & $0.038 \mathrm{a}$ & $0.357 \mathrm{a}$ & $1.013 a$ & $1.731 \mathrm{a}$ & $0.039 a$ & $0.325 b$ & $0.875 b$ & $1.366 \mathrm{~b}$ & $0.031 \mathrm{~b}$ & $0.294 b$ & $0.844 \mathrm{~b}$ & $1.194 \mathrm{~b}$ & $0.003 b$ & $0.248 b$ & $0.805 b$ & $1.102 b$ \\
\hline 2 & $0.039 a$ & $0.314 \mathrm{c}$ & $0.881 \mathrm{c}$ & $1.227 \mathrm{c}$ & $0.031 \mathrm{c}$ & $0.283 c$ & $0.825 c$ & $1.093 \mathrm{c}$ & $0.003 \mathrm{~b}$ & $0.267 \mathrm{c}$ & $0.772 \mathrm{c}$ & $1.053 \mathrm{c}$ & $0.028 \mathrm{~b}$ & $0.204 c$ & $0.717 \mathrm{c}$ & $0.944 c$ \\
\hline 3 & $0.033 \mathrm{~b}$ & $0.282 \mathrm{~d}$ & $0.760 \mathrm{~d}$ & $1.102 \mathrm{~d}$ & $0.028 d$ & $0.253 \mathrm{~d}$ & $0.733 \mathrm{~d}$ & $1.002 \mathrm{~d}$ & $0.027 c$ & $0.214 d$ & $0.705 d$ & $0.993 \mathrm{~d}$ & $0.025 c$ & $0.184 d$ & $0.599 \mathrm{~d}$ & $0.893 d$ \\
\hline 4 & $0.029 c$ & $0.263 \mathrm{e}$ & $0.618 \mathrm{e}$ & $0.962 \mathrm{e}$ & $0.026 \mathrm{~d}$ & $0.224 \mathrm{e}$ & $0.576 \mathrm{e}$ & $0.896 \mathrm{e}$ & $0.025 \mathrm{~d}$ & $0.197 \mathrm{e}$ & $0.544 \mathrm{e}$ & $0.866 \mathrm{e}$ & $0.022 \mathrm{~d}$ & $0.167 \mathrm{e}$ & $0.474 \mathrm{e}$ & $0.736 \mathrm{e}$ \\
\hline
\end{tabular}

Mean with different alphabets in a column differed significantly as per Tukey's Multiple Range Test (TMRT) $(\mathrm{P}<0.05)$.

Table 5. Leaf Dry weight (g/plant) of Green gram plants treated with various concentrations of individual and combined weed residues.

\begin{tabular}{|c|c|c|c|c|c|c|c|c|c|c|c|c|c|c|c|c|}
\hline \multirow{3}{*}{$\begin{array}{c}\text { Weed } \\
\text { Residues } \\
(\%)\end{array}$} & \multicolumn{4}{|c|}{ E. colona } & \multicolumn{4}{|c|}{ C. viscosa } & \multicolumn{4}{|c|}{ A. baccifera } & \multicolumn{4}{|c|}{ Combined weeds } \\
\hline & \multicolumn{4}{|c|}{ DAS } & \multicolumn{4}{|c|}{ DAS } & \multicolumn{4}{|c|}{ DAS } & \multicolumn{4}{|c|}{ DAS } \\
\hline & 15 & 30 & 45 & 60 & 15 & 30 & 45 & 60 & 15 & 30 & 45 & 60 & 15 & 30 & 45 & 60 \\
\hline $\mathrm{C}$ & $0.083 a$ & $1.073 a$ & $4.027 \mathrm{a}$ & $4.429 \mathrm{a}$ & $0.083 a$ & $1.073 a$ & $4.027 \mathrm{a}$ & $4.429 \mathrm{a}$ & $0.083 a$ & $1.073 a$ & $3.937 a$ & $4.429 a$ & $0.083 a$ & $1.073 a$ & $4.027 \mathrm{a}$ & $4.429 \mathrm{a}$ \\
\hline 1 & $0.090 \mathrm{a}$ & $1.184 a$ & $4.145 a$ & $4.633 a$ & $0.080 \mathrm{a}$ & $1.044 a$ & $3.826 a$ & $4.222 \mathrm{a}$ & $0.077 \mathrm{a}$ & $1.030 \mathrm{a}$ & $3.528 \mathrm{~b}$ & $4.058 \mathrm{a}$ & $0.075 b$ & $1.001 \mathrm{a}$ & $3.326 \mathrm{~b}$ & $3.888 \mathrm{~b}$ \\
\hline 2 & $0.079 \mathrm{~b}$ & $1.039 \mathrm{~b}$ & $3.698 b$ & $4.200 c$ & $0.076 \mathrm{~b}$ & $1.013 \mathrm{~b}$ & $3.548 b$ & $3.888 b$ & $0.074 \mathrm{~b}$ & $0.957 \mathrm{~b}$ & $3.382 \mathrm{c}$ & $3.669 \mathrm{~b}$ & $0.069 c$ & $0.941 \mathrm{~b}$ & $3.063 c$ & $3.273 c$ \\
\hline 3 & $0.072 \mathrm{c}$ & $1.010 \mathrm{c}$ & $3.325 c$ & $3.822 \mathrm{~d}$ & $0.069 c$ & $0.871 \mathrm{c}$ & $3.133 c$ & $3.450 \mathrm{c}$ & $0.067 \mathrm{c}$ & $0.793 \mathrm{c}$ & $2.863 \mathrm{c}$ & $3.212 \mathrm{c}$ & $0.057 \mathrm{~d}$ & $0.713 c$ & $2.326 \mathrm{~d}$ & $3.050 \mathrm{~d}$ \\
\hline 4 & $0.070 \mathrm{~d}$ & $0.893 \mathrm{~d}$ & $3.078 \mathrm{~d}$ & $3.656 \mathrm{e}$ & $0.062 \mathrm{~d}$ & $0.813 \mathrm{~d}$ & $2.848 \mathrm{~d}$ & $3.150 \mathrm{~d}$ & $0.059 \mathrm{~d}$ & $0.717 \mathrm{~d}$ & $2.427 \mathrm{~d}$ & $2.662 \mathrm{e}$ & $0.052 \mathrm{e}$ & $0.574 \mathrm{~d}$ & $2.027 \mathrm{e}$ & $2.519 \mathrm{e}$ \\
\hline
\end{tabular}

Mean with different alphabets in a column differed significantly as per Tukey's Multiple Range Test (TMRT) $(\mathrm{P}<0.05)$. 


\section{REFERENCES}

Bartariya, G., A. Saxena, J.N. Srivastva and G.P. Satsangi, (2005). Allelopathic potential of Cyperus rotundus $\mathrm{L}$. on germination and seedling growth of Oryza sativa L. Allelopathy J 6(2): 353-358.

Challa, P. and V. Ravindra, (1998). Allelopathic effects of major weeds on vegetable crops. Allelopathy J 5: 89-92.

Dos Santosh, W.D., M.L.L. Ferrarese, A. Finger, A.C.N. Teixeira and 0. Ferrarese-Filho, (2004). Lignification and related enzymes in Glycine max root growth- inhibition by ferulic acid. $J$. Chem. Ecol 30: 1199-1208.

Gniazdowska, A. and R. Bogatek, (2005). Allelopathic interactions between plants. Multisite action of allelochemicals Acta Physiologiae Plantarum 27: 395-407.

Hamayun, M., F. Hussain, S. Afzal and N. Ahmad, (2005). Allelopathic effects of Cyperus rotunds and Echinochloa crus-galli on seed grermination and plumule and radical growth in maize (Zea mays L.). Pak. J. Weed Sci. Res 11: 81-84.

Kumar, V., C.D. Brainerd and R.R. Blender, (2009). Suppression of Powell Amaranth (Amaranthus powellii) by Buckwheat Residues: Role of Allelopathy. Weed Sci 57(1): 66-73.

Patrick, Z.A. and L.W. Koch, (1963). The adverse influence of phytotoxic substances from decomposing plant residues on resistance of tobacco to black root rot. Canad. J. Bot 41: 747758.

Qasem, J.R. and T.R. Hill, (1989). Possible role of allelopathy in competition between tomato, Senecio vulgaris L. Chenopodium album L. Weed Res 29: 349-356.

Quayyam, H.A., A.V. Malik, D.M. Leach and C. Gottardo, (2000). Growth inhibitory effects of nutgrass (Cyperus rotundus) on rice (Oryza sativa). J. Chem. Eco 26: 2221-2231.

Rajashekhara, R.B.K., T.N. Shivananda, J. Syamasundara and R. Siddaramappa, (2007). Effects of allelochemicals from selected carbon sources under flooded soil conditions on histological abnormalities induced in rice (Oryza sativa L.) roots. Allelopath. J. 20(2): 347353.

Sannigrahi, A.K. and S. Chakrabortty, (2005). Allelopathic effects of weeds on germination and seedling growth of tomato. Allelopathy $J$ 16(2): 289-294

Tanveer, A., M. Tahir, M.A. Nadeem, M. Younis, A. Aziz and M. Yaseen, (2008). Allelopathic effects of Xanthium strumarium L. on seed germination and seedling growth of crops. Allelopath. J 21(2): 317-328

Venkateshwarlu, G., V. Ravindra and C. Prabha, (2001). Mangiferin: an allelopathin from mango (Mangifera indica L.) leaves. Allelopathy J 8: 221-224. 